\section{Likelihood Gradient Evaluation Using Square-Root Covariance Filters}

\author{
M.V. Kulikova
}

\begin{abstract}
Using the array form of numerically stable square-root implementation methods for Kalman filtering formulas, we construct a new square-root algorithm for the log-likelihood gradient (score) evaluation. This avoids the use of the conventional Kalman filter with its inherent numerical instabilities and improves the robustness of computations against roundoff errors. The new algorithm is developed in terms of covariance quantities and based on the "condensed form" of the array square-root filter.
\end{abstract}

Index Terms - identification, maximum likelihood estimation, gradient methods, Kalman filtering, numerical stability.

\section{INTRODUCTION}

Consider the discrete-time linear stochastic system

$$
\begin{aligned}
x_{k} & =F_{k} x_{k-1}+G_{k} w_{k}, \\
z_{k} & =H_{k} x_{k}+v_{k}, \quad k=1, \ldots, N,
\end{aligned}
$$

where $x_{k} \in \mathbb{R}^{n}$ and $z_{k} \in \mathbb{R}^{m}$ are, respectively, the state and the measurement vectors; $k$ is a discrete time, i.e. $x_{k}$ means $x\left(t_{k}\right)$. The noises $w_{k} \in \mathbb{R}^{q}, v_{k} \in \mathbb{R}^{m}$ and the initial state $x_{0} \sim \mathcal{N}\left(\bar{x}_{0}, \Pi_{0}\right)$ are taken from mutually independent Gaussian distributions with zero mean and covariance matrices $Q_{k}$ and $R_{k}$, respectively, i.e. $w_{k} \sim \mathcal{N}\left(0, Q_{k}\right)$, $v_{k} \sim \mathcal{N}\left(0, R_{k}\right)$. Additionally, system (1), (2) is parameterized by a vector of unknown system parameters $\theta \in \mathbb{R}^{p}$, which needs to be estimated. This means that the entries of the matrices $F_{k}, G_{k}, H_{k}$, $Q_{k}, R_{k}$ and $\Pi_{0}$ are functions of $\theta \in \mathbb{R}^{p}$. However, for the sake of simplicity we will suppress the corresponding notations below, i.e instead of $F_{k}(\theta), G_{k}(\theta), H_{k}(\theta), Q_{k}(\theta), R_{k}(\theta)$ and $\Pi_{0}(\theta)$ we will write $F_{k}, G_{k}, H_{k}, Q_{k}, R_{k}$ and $\Pi_{0}$.

Solving the parameter estimation problem by the method of maximum likelihood requires the maximization of the likelihood function (LF) with respect to unknown system parameters. It is often done by using a gradient approach where the computation of the likelihood gradient (LG) is necessary. For the state-space system (1), (2) the negative $\log \mathrm{LF}$ is given as [1]:

$$
L_{\theta}\left(Z_{1}^{N}\right)=\frac{1}{2} \sum_{k=1}^{N}\left\{\frac{m}{2} \ln (2 \pi)+\ln \left(\operatorname{det} R_{e, k}\right)+e_{k}^{T} R_{e, k}^{-1} e_{k}\right\}
$$

where $Z_{1}^{N}=\left[z_{1}, \ldots, z_{N}\right]$ is $N$-step measurement history and $e_{k}$ are the innovations, generated by the discrete-time Kalman filter (KF), with zero mean and covariance matrix $R_{e, k}$. They are $e_{k}=z_{k}-$ $H_{k} \hat{x}_{k \mid k-1}$ and $R_{e, k}=H_{k} P_{k \mid k-1} H_{k}^{T}+R_{k}$, respectively. The KF defines the one-step ahead predicted state estimate $\hat{x}_{k \mid k-1}$ and the one-step predicted error covariance matrix $P_{k \mid k-1}$.

Straight forward differentiation of the KF equations is a direct approach to the Log LG evaluation, known as a "score". This leads to a set of $p$ vector equations, known as the filter sensitivity equations, for computing $\partial \hat{x}_{k \mid k-1} / \partial \theta$, and a set of $p$ matrix equations, known as the Riccati-type sensitivity equations, for computing $\partial P_{k \mid k-1} / \partial \theta$.

Consequently, the main disadvantage of the standard approach is the problem of numerical instability of the conventional KF, i.e divergence due to the lack of reliability of the numerical algorithm. Solution of the matrix Riccati equation is a major cause of numerical difficulties in the conventional KF implementation, from the

Manuscript received September 14, 2007; revised May 9, 2008.

M.V. Kulikova is with the School of Computational and Applied Mathematics, University of the Witwatersrand, South Africa; Email: Maria.Kulikova@wits.ac.za. standpoint of computational load as well as from the standpoint of computational errors [2].

The alternative approach can be found in, so-called, squareroot filtering algorithms. It is well known that numerical solution of the Riccati equation tends to be more robust against roundoff errors if Cholesky factors or modified Cholesky factors (such as the $U^{T} D U$-algorithms [3]) of the covariance matrix are used as the dependent variables. The resulting KF implementation methods are called square-root filters (SRF). They are now generally preferred for practical use [2], [4], [5]. For more insights about numerical properties of different KF implementation methods we refer to the celebrated paper of Verhaegen and Van Dooren [6].

Increasingly, the preferred form for algorithms in many fields is now the array form [7]. Several useful SRF algorithms for KF formulas formulated in the array form have been recently proposed by Park and Kailath [8]. For this implementations the reliability of the filter estimates is expected to be better because of the use of numerically stable orthogonal transformations for each recursion step. Apart from numerical advantages, array SRF algorithms appear to be better suited to parallel and to very large scale integration (VLSI) implementations [8], [9].

The development of numerically stable implementation methods for $\mathrm{KF}$ formulas has led to the hope that the Log LG (with respect to unknown system parameters) might be computed more accurately. For this problem, a number of questions arise:

- Is it possible to extend reliable array SRF algorithms to the case of the Log LG evaluation?

- If such methods exist, will they inherit the advantages from the source filtering implementations? In particular, will they improve the robustness of the computations against roundoff errors compared to the conventional KF technique? The question about suitability for parallel implementation is beyond the scope of this paper.

The first attempt to answer these questions belongs to Bierman et al. [10]. The authors used the square-root information filter, developed by Dyer and McReynolds [11] and later extended by Bierman [3], as a source filter implementation and constructed the method for score evaluation. The algorithm was developed in the form of measurement and time updates. However, the accuracy of the proposed method has not been investigated.

In contrast to the main result of [10], we focus on the dual class of KF implementation methods (that is the class of covariance-type methods) and discuss the efficient Log LG evaluation in square-root covariance filters. More precisely, we consider the array form of the square-root covariance filter eSRCF introduced in [8]. The purpose of this paper is to design the method for the Log LG evaluation in terms of the square-root covariance variables, i.e. in terms of the quantities that appear naturally in the eSRCF. This avoids the use of the conventional $\mathrm{KF}$ with its inherent numerical instabilities and gives us an opportunity to improve the robustness of the Log LG computation against roundoff errors.

\section{Extended Square-Root Covariance FiLter}

To achieve our goal, we are first going to present the extended square-root covariance filter (eSRCF), proposed in [8], and second, we will derive the expression for the Log LG evaluation in terms of the variables that are generated by the eSRCF implementation.

Notations to be used: For the sake of simplicity, we denote the one-step predicted state estimate as $\hat{x}_{k}$ and the one-step predicted error covariance matrix as $P_{k}$. We use Cholesky decomposition of the form $P_{k}=P_{k}^{T / 2} P_{k}^{1 / 2}$, where $P_{k}^{1 / 2}$ is an upper triangular matrix. Similarly, we define $R_{k}^{1 / 2}, Q_{k}^{1 / 2}, R_{e, k}^{1 / 2}$. For convenience we will write 


$$
\begin{aligned}
& Q_{k}\left[\begin{array}{cc|c||cc|c}
R_{k}^{1 / 2} & 0 & -R_{k}^{-T / 2} z_{k} & \partial_{\theta_{i}} R_{k}^{1 / 2} & 0 \\
P_{k}^{1 / 2} H_{k}^{T} & P_{k}^{1 / 2} F_{k}^{T} & P_{k}^{-T / 2} \hat{x}_{k} & \partial_{\theta_{i}}\left(P_{k}^{1 / 2} H_{k}^{T}\right) & \partial_{\theta_{i}}\left(P_{k}^{1 / 2} F_{k}^{T}\right) \\
0 & Q_{k}^{1 / 2} G_{k}^{T} & 0 & 0 & \partial_{\theta_{i}}\left(Q_{k}^{1 / 2} G_{k}^{T}\right) & \partial_{\theta_{i}}\left(-R_{k}^{-T / 2} z_{k}\right) \\
\partial_{\theta_{i}}\left(P_{k}^{-T / 2} \hat{x}_{k}\right) \\
0
\end{array}\right] \\
& =\left[\begin{array}{cc|c||cc|c}
R_{e, k}^{1 / 2} & \bar{K}_{p, k}^{T} & -\bar{e}_{k} & X_{i} & Y_{i} & M_{i} \\
0 & P_{k+1}^{1 / 2} & P_{k+1}^{-T / 2} \hat{x}_{k+1} & N_{i} & V_{i} & W_{i} \\
0 & 0 & \gamma_{k} & B_{i} & K_{i} & T_{i}
\end{array}\right] \text {. }
\end{aligned}
$$

$A^{-1 / 2}=\left(A^{1 / 2}\right)^{-1}, A^{-T / 2}=\left(A^{-1 / 2}\right)^{T}$ and $\partial_{\theta_{i}} A$ implies the partial derivative of the matrix $A$ with respect to the $i$ th component of $\theta$, i.e $\partial A / \partial \theta_{i}$.

In this paper, we deal with the "condensed form' 1 of the eSRCF [8]: Assume that $R_{k}>0$. Given $\Pi_{0}^{1 / 2}$ and $\Pi_{0}^{-T / 2} \bar{x}_{0}$, recursively update $P_{k}^{1 / 2}$ and $P_{k}^{-T / 2} \hat{x}_{k}$ as follows:

$$
\begin{array}{r}
Q_{k}\left[\begin{array}{cc|c}
R_{k}^{1 / 2} & 0 & -R_{k}^{-T / 2} z_{k} \\
P_{k}^{1 / 2} H_{k}^{T} & P_{k}^{1 / 2} F_{k}^{T} & P_{k}^{-T / 2} \hat{x}_{k} \\
0 & Q_{k}^{1 / 2} G_{k}^{T} & 0
\end{array}\right] \\
=\left[\begin{array}{cc|c}
R_{e, k}^{1 / 2} & \bar{K}_{p, k}^{T} & -\bar{e}_{k} \\
0 & P_{k+1}^{1 / 2} & P_{k+1}^{-T / 2} \hat{x}_{k+1} \\
0 & 0 & \gamma_{k}
\end{array}\right]
\end{array}
$$

where $Q_{k}$ is any orthogonal rotation that upper-triangularizes the first two (block) columns of the matrix on the left-hand side of 3 ; $\bar{K}_{p, k}=F_{k} P_{k} H_{k}^{T} R_{e, k}^{-1 / 2}$ and $\bar{e}_{k}=R_{e, k}^{-T / 2} e_{k}$.

One can easily obtain the expression for the negative Log LF in terms of the eSRCF variables:

$$
L_{\theta}\left(Z_{1}^{N}\right)=\frac{1}{2} \sum_{k=1}^{N}\left\{\frac{m}{2} \ln (2 \pi)+2 \ln \left(\operatorname{det} R_{e, k}^{1 / 2}\right)+\bar{e}_{k}^{T} \bar{e}_{k}\right\} .
$$

Let $\theta=\left[\theta_{1}, \ldots, \theta_{p}\right]$ denote the vector of parameters with respect to which the likelihood function is to be differentiated. Then from (4), we have

$$
\partial_{\theta_{i}} L_{\theta}\left(Z_{1}^{N}\right)=\sum_{k=1}^{N}\left\{\partial_{\theta_{i}}\left[\ln \left(\operatorname{det} R_{e, k}^{1 / 2}\right)\right]+\frac{1}{2} \partial_{\theta_{i}}\left[\bar{e}_{k}^{T} \bar{e}_{k}\right]\right\} .
$$

Taking into account that the matrix $R_{e, k}^{1 / 2}$ is upper triangular, we derive

$$
\begin{aligned}
\partial_{\theta_{i}}\left[\ln \left(\operatorname{det} R_{e, k}^{1 / 2}\right)\right] & =\partial_{\theta_{i}}\left[\sum_{j=1}^{m} \ln \left(r_{e, k}^{j j}\right)\right]=\sum_{j=1}^{m}\left[\frac{1}{r_{e, k}^{j j}} \cdot \partial_{\theta_{i}} r_{e, k}^{j j}\right] \\
& =\operatorname{tr}\left[R_{e, k}^{-1 / 2} \cdot \partial_{\theta_{i}} R_{e, k}^{1 / 2}\right], \quad i=1, \ldots, p
\end{aligned}
$$

where the $r_{e, k}^{j j}, j=1, \ldots, m$ denote the diagonal elements of the matrix $R_{e, k}^{1 / 2}$.

Substitution of (6) into 5 yields the result that we are looking for

$$
\partial_{\theta_{i}} L_{\theta}\left(Z_{1}^{N}\right)=\sum_{k=1}^{N}\left\{\operatorname{tr}\left[R_{e, k}^{-1 / 2} \cdot \partial_{\theta_{i}} R_{e, k}^{1 / 2}\right]+\bar{e}_{k}^{T} \cdot \partial_{\theta_{i}} \bar{e}_{k}\right\} \text {. }
$$

Ultimately, our problem is to compute Log LG (7) by using the eSRCF equation (3). Before we come to the main result of this paper, there are a few points to be considered. As can be seen from (7), the elements $\bar{e}_{k}$ and $R_{e, k}^{1 / 2}$ involved in the Log LG evaluation are obtained from the underlying filtering algorithm directly, i.e. from 3 . No additional computations are needed. Hence, our aim is to explain

\footnotetext{
${ }^{1}$ The "condensed form" of filtering algorithms refers to the case when implementation method for the KF formulas is not divided into the measurement and time updates.
}

how the last two terms in the Log LG expression, $\partial_{\theta_{i}} \bar{e}_{k}$ and $\partial_{\theta_{i}} R_{e, k}^{1 / 2}$, can be computed using quantities available from eSRCF (3).

\section{Suggested Square-Root Method for Score EVALUATION}

We can now prove the following result.

Theorem 1: Let the entries of the matrices $F_{k}, G_{k}, H_{k}, Q_{k}, R_{k}$, $\Pi_{0}$ describing the linear discrete-time stochastic system (1), (2) be differentiable functions of a parameter $\theta \in \mathbb{R}^{p}$. Then in order to compute the Log LF and its gradient (with respect to unknown system parameter $\theta$ ) the eSRCF, which is used to filter the data, needs to be extended as follows. Assume that $R_{k}>0$. Given the initial values $\Pi_{0}^{1 / 2}, \Pi_{0}^{-T / 2} \bar{x}_{0}$ and $\partial_{\theta_{i}} \Pi_{0}^{1 / 2}, \partial_{\theta_{i}}\left(\Pi_{0}^{-T / 2} \bar{x}_{0}\right)$, recursively update $P_{k}^{1 / 2}, P_{k}^{-T / 2} \hat{x}_{k}$ and $\partial_{\theta_{i}} P_{k}^{1 / 2}, \partial_{\theta_{i}}\left(P_{k}^{-T / 2} \hat{x}_{k}\right)$ as follows:

I. Replace the eSRCF equation (3) by (8) where $Q_{k}$ is any orthogonal rotation that upper-triangularizes the first two (block) columns of the matrix on the left-hand side of (8).

II. Having computed the elements of the right-hand side matrix in (8), calculate for each $\theta_{i}$ :

$$
\begin{gathered}
{\left[\begin{array}{cc}
\partial_{\theta_{i}} R_{e, k}^{1 / 2} & \partial_{\theta_{i}} \bar{K}_{p, k}^{T} \\
0 & \partial_{\theta_{i}} P_{k+1}^{1 / 2}
\end{array}\right]=\left[\bar{L}_{i}^{T}+D_{i}+\bar{U}_{i}\right]\left[\begin{array}{cc}
R_{e, k}^{1 / 2} & \bar{K}_{p, k}^{T} \\
0 & P_{k+1}^{1 / 2}
\end{array}\right]} \\
{\left[\begin{array}{c}
-\partial_{\theta_{i}} \bar{e}_{k} \\
\partial_{\theta_{i}}\left(P_{k+1}^{-T / 2} \hat{x}_{k+1}\right)
\end{array}\right]=\left[\bar{L}_{i}^{T}-\bar{L}_{i}\right]\left[\begin{array}{c}
-\bar{e}_{k} \\
P_{k+1}^{-T / 2} \hat{x}_{k+1}
\end{array}\right]} \\
+\left[\begin{array}{cc}
R_{e, k}^{1 / 2} & \bar{K}_{p, k}^{T} \\
0 & P_{k+1}^{1 / 2}
\end{array}\right]
\end{gathered}
$$

where $\bar{L}_{i}, D_{i}$ and $\bar{U}_{i}$ are strictly lower triangular, diagonal and strictly upper triangular parts of the following matrix product:

$$
\left[\begin{array}{cc}
X_{i} & Y_{i} \\
N_{i} & V_{i}
\end{array}\right]\left[\begin{array}{cc}
R_{e, k}^{-1 / 2} & -R_{e, k}^{-1 / 2} \bar{K}_{p, k}^{T} P_{k+1}^{-1 / 2} \\
0 & P_{k+1}^{-1 / 2}
\end{array}\right]=\bar{L}_{i}+D_{i}+\bar{U}_{i} .
$$

III. Having determined $\bar{e}_{k}, R_{e, k}^{1 / 2}$ and $\partial_{\theta_{i}} \bar{e}_{k}, \partial_{\theta_{i}} R_{e, k}^{1 / 2}$ compute Log LF (4) and Log LG (7).

Proof: As discussed earlier, the main difficulty in score evaluation (7) is to define $\partial_{\theta_{i}} R_{e, k}^{1 / 2}$ and $\partial_{\theta_{i}} \bar{e}_{k}$ from the underlying filter, i.e. from (3). We divide the proof into two parts, first proving (9) for the $\partial_{\theta_{i}} R_{e, k}^{1 / 2}$ evaluation and then validating (10) for $\partial_{\theta_{i}} \bar{e}_{k}$.

Part I. Our goal is to express $\partial_{\theta_{i}} R_{e, k}^{1 / 2}$ in terms of the variables that appear naturally in the eSRCF implementation. First, we can note that the eSRCF transformation in 3 has a form

$$
Q A=B
$$

where $A$ is a rectangular matrix, and $Q$ is an orthogonal transformation that block upper-triangularizes $B$. If matrix $A$ is square and 
invertible, then given the matrix of derivatives $A_{\theta}^{\prime}=\frac{d a_{i j}}{d \theta}$ we can compute $B_{\theta}^{\prime}$ as follows [10]:

$$
B_{\theta}^{\prime}=\left[L^{T}+D+U\right] B
$$

where $L, D$ and $U$ are, respectively, strictly lower triangular, diagonal and strictly upper triangular parts of the matrix $Q A_{\theta}^{\prime} B^{-1}$.

However, this idea cannot be applied to the eSRCF because the matrix to be triangularized, i.e. the first two (block) columns of the matrix on the left-hand side of (3), is not square and, hence, not invertible. By using the pseudoinversion (Moore-Penrose inversion) we avoid this obstacle and generalize the scheme of computations to the case of eSRCF (3).

To begin constructing the method for score evaluation, we augment the matrix to be triangularized by $q$ columns of zeros. Hence, we obtain

$$
Q_{k}\left[\begin{array}{cc|c}
R_{k}^{1 / 2} & 0 & 0 \\
P_{k}^{1 / 2} H_{k}^{T} & P_{k}^{1 / 2} F_{k}^{T} & 0 \\
0 & Q_{k}^{1 / 2} G_{k}^{T} & 0
\end{array}\right]=\left[\begin{array}{cc|c}
R_{e, k}^{1 / 2} & \bar{K}_{p, k}^{T} & 0 \\
0 & P_{k+1}^{1 / 2} & 0 \\
0 & 0 & 0
\end{array}\right] .
$$

The matrices in (13) have dimensions $(m+n+q) \times(m+n+q)$. For the sake of simplicity, we denote the left-hand side and the righthand side matrices of (13) as $A_{k}$ and $B_{k}$, respectively. Then, by differentiating (13) with respect to the components of $\theta$, we obtain

$$
\partial_{\theta_{i}} Q_{k} \cdot A_{k}+Q_{k} \cdot \partial_{\theta_{i}} A_{k}=\partial_{\theta_{i}} B_{k} .
$$

Multiplication both sides of (14) by the pseudoinverse matrix $B_{k}^{+}$ yields

$$
\begin{aligned}
\partial_{\theta_{i}} B_{k} \cdot B_{k}^{+} & =\partial_{\theta_{i}} Q_{k}\left(A_{k} B_{k}^{+}\right)+Q_{k} \cdot \partial_{\theta_{i}} A_{k} \cdot B_{k}^{+} \\
& =\partial_{\theta_{i}} Q_{k}\left(Q_{k}^{T} B_{k} B_{k}^{+}\right)+\left(Q_{k} \cdot \partial_{\theta_{i}} A_{k}\right) B_{k}^{+} .
\end{aligned}
$$

One can easily obtain the explicit expression for $B_{k}^{+}$:

$$
B_{k}^{+}=\left[\begin{array}{ccc}
R_{e, k}^{-1 / 2} & -R_{e, k}^{-1 / 2} \bar{K}_{p, k}^{T} P_{k+1}^{-1 / 2} & 0 \\
0 & P_{k+1}^{-1 / 2} & 0 \\
0 & 0 & 0
\end{array}\right]
$$

By using $(8)$, we replace $Q_{k} \cdot \partial_{\theta_{i}} A_{k}$ in 15 by the quantities already computed. Then, taking into account (16), we derive the equation for the $(m+n) \times(m+n)$ main block of the matrix $B_{k}$ :

$$
\begin{array}{r}
{\left[\begin{array}{cr}
\partial_{\theta_{i}} R_{e, k}^{1 / 2} & \partial_{\theta_{i}} \bar{K}_{p, k}^{T} \\
0 & \partial_{\theta_{i}} P_{k+1}^{1 / 2}
\end{array}\right]\left[\begin{array}{cr}
R_{e, k}^{1 / 2} & \bar{K}_{p, k}^{T} \\
0 & P_{k+1}^{1 / 2}
\end{array}\right]^{-1}=\left[\partial_{\theta_{i}} Q_{k} \times Q_{k}^{T}\right]_{m+n}} \\
+\left[\begin{array}{c}
X_{i} Y_{i} \\
N_{i} V_{i}
\end{array}\right]\left[\begin{array}{cc}
R_{e, k}^{1 / 2} & \bar{K}_{p, k}^{T} \\
0 & P_{k+1}^{1 / 2}
\end{array}\right]^{-1}
\end{array}
$$

where $\left[\partial_{\theta_{i}} Q_{k} \cdot Q_{k}^{T}\right]_{m+n}$ denotes the $(m+n) \times(m+n)$ main block of the matrix $\partial_{\theta_{i}} Q_{k} \cdot Q_{k}^{T}$.

As discussed in [10], the matrix $\partial_{\theta_{i}} Q_{k} \cdot Q_{k}^{T}$ is skew symmetric and, hence, can be represented in the form $\bar{L}^{T}-\bar{L}$ where $\bar{L}$ is strictly lower triangular.

Now, let us consider matrix equation (17). As can be seen, the matrix on the left-hand side of (17) is block upper triangular. Thus, the strictly lower triangular part of the matrix $\left[\partial_{\theta_{i}} Q_{k} \cdot Q_{k}^{T}\right]_{m+n}$ must exactly cancel the strictly lower triangular part of the second term on the right-hand side of 177. In other words, if

$$
\left[\begin{array}{cc}
X_{i} & Y_{i} \\
N_{i} & V_{i}
\end{array}\right]\left[\begin{array}{cc}
R_{e, k}^{1 / 2} & \bar{K}_{p, k}^{T} \\
0 & P_{k+1}^{1 / 2}
\end{array}\right]^{-1}=\bar{L}_{i}+D_{i}+\bar{U}_{i}
$$

then

$$
\left[\partial_{\theta_{i}} Q_{k} \cdot Q_{k}^{T}\right]_{m+n}=\bar{L}_{i}^{T}-\bar{L}_{i} .
$$

Substitution of (18) into (17) leads to the result

$$
\left[\begin{array}{cc}
\partial_{\theta_{i}} R_{e, k}^{1 / 2} & \partial_{\theta_{i}} \bar{K}_{p, k}^{T} \\
0 & \partial_{\theta_{i}} P_{k+1}^{1 / 2}
\end{array}\right]=\left[\bar{L}_{i}^{T}+D_{i}+\bar{U}_{i}\right]\left[\begin{array}{cc}
R_{e, k}^{1 / 2} & \bar{K}_{p, k}^{T} \\
0 & P_{k+1}^{1 / 2}
\end{array}\right] \text {. }
$$

Formulas (19) and 18 are, in fact, equations 9 and 111 of the proposed method for score evaluation. The theorem is half proved.

Part II. We need to verify (10). By differentiating the last equation of the eSRCF with respect to the components of $\theta$

$$
Q_{k}\left[\begin{array}{c}
-R_{k}^{-T / 2} z_{k} \\
P_{k}^{-T / 2} \hat{x}_{k} \\
0
\end{array}\right]=\left[\begin{array}{c}
-\bar{e}_{k} \\
P_{k+1}^{-T / 2} \hat{x}_{k+1} \\
\gamma_{k}
\end{array}\right]
$$

we obtain

$$
\begin{array}{r}
{\left[\begin{array}{c}
-\partial_{\theta_{i}} \bar{e}_{k} \\
\partial_{\theta_{i}}\left(P_{k+1}^{-T / 2} \hat{x}_{k+1}\right) \\
\partial_{\theta_{i}} \gamma_{k}
\end{array}\right]=\partial_{\theta_{i}} Q_{k} \cdot Q_{k}^{T} \cdot Q_{k}\left[\begin{array}{c}
-R_{k}^{-T / 2} z_{k} \\
P_{k}^{-T / 2} \hat{x}_{k} \\
0
\end{array}\right]} \\
+Q_{k}\left[\begin{array}{c}
-\partial_{\theta_{i}}\left(R_{k}^{-T / 2} z_{k}\right) \\
\partial_{\theta_{i}}\left(P_{k}^{-T / 2} \hat{x}_{k}\right) \\
0
\end{array}\right] .
\end{array}
$$

Next, we replace the last term in (20) with the quantities already computed and collected in the right-hand side matrix of (8). Furthermore, it is useful to note that the element $\partial_{\theta_{i}} \gamma_{k}$ is of no interest here. These two steps give us

$$
\begin{aligned}
{\left[\begin{array}{c}
-\partial_{\theta_{i}} \bar{e}_{k} \\
\partial_{\theta_{i}}\left(P_{k+1}^{-T / 2} \hat{x}_{k+1}\right)
\end{array}\right] } & =\left[\partial_{\theta_{i}} Q_{k} \cdot Q_{k}^{T}\right]_{m+n}\left[\begin{array}{c}
-\bar{e}_{k} \\
P_{k+1}^{-T / 2} \hat{x}_{k+1}
\end{array}\right] \\
& +\left[\partial_{\theta_{i}} Q_{k} \cdot Q_{k}^{T}\right]_{\text {col: last } q}^{\text {row: } 1: m+n} \gamma_{k}+\left[\begin{array}{c}
M_{i} \\
W_{i}
\end{array}\right]
\end{aligned}
$$

where $\left[\partial_{\theta_{i}} Q_{k} \cdot Q_{k}^{T}\right]_{\text {col: last } q}^{\text {row: } 1: m+n}$ stands for the $(m+n) \times q$ matrix composed of the entries that are located at the intersections of the last $q$ columns with the first $m+n$ rows of $\partial_{\theta_{i}} Q_{k} \cdot Q_{k}^{T}$.

Taking into account (18), from the equation above we obtain

$$
\begin{aligned}
{\left[\begin{array}{c}
-\partial_{\theta_{i}} \bar{e}_{k} \\
\partial_{\theta_{i}}\left(P_{k+1}^{-T / 2} \hat{x}_{k+1}\right)
\end{array}\right] } & =\left[\bar{L}_{i}^{T}-\bar{L}_{i}\right]\left[\begin{array}{c}
-\bar{e}_{k} \\
P_{k+1}^{-T / 2} \hat{x}_{k+1}
\end{array}\right] \\
& +\left[\partial_{\theta_{i}} Q_{k} \cdot Q_{k}^{T}\right]_{\text {col: last } q}^{\text {row: } 1: m+n} \gamma_{k}+\left[\begin{array}{c}
M_{i} \\
W_{i}
\end{array}\right]
\end{aligned}
$$

where $\bar{L}_{i}$ is strictly lower triangular part of the matrix in 11.

Since $\partial_{\theta_{i}} Q_{k} \cdot Q_{k}^{T}$ is skew symmetric, we can write down

$$
\left[\partial_{\theta_{i}} Q_{k} \cdot Q_{k}^{T}\right]_{\text {col: last } q}^{\text {row: } 1: m+n}=-\left[\left[\partial_{\theta_{i}} Q_{k} \cdot Q_{k}^{T}\right]_{\text {col: } 1: m+n}^{\text {row: last } q}\right]^{T}
$$

where $\left[\partial_{\theta_{i}} Q_{k} \cdot Q_{k}^{T}\right]_{\text {col: } 1: m+n}^{\text {row: last } q}$ stands for the $q \times(m+n)$ matrix composed of the entries that are located at the intersections of the last $q$ rows with the first $(m+n)$ columns of $\partial_{\theta_{i}} Q_{k} \cdot Q_{k}^{T}$.

To evaluate the right-hand side of (23), we return to 15 and write it in the matrix form:

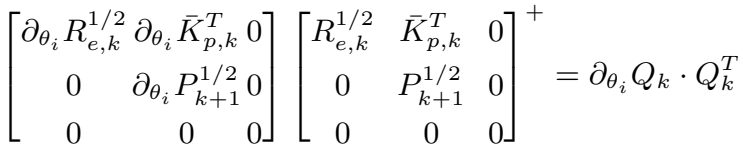

$$
\begin{aligned}
& +\left[\begin{array}{ccc}
X_{i} & Y_{i} & 0 \\
N_{i} & V_{i} & 0 \\
B_{i} & K_{i} & 0
\end{array}\right]\left[\begin{array}{ccc}
R_{e, k}^{1 / 2} & \bar{K}_{p, k}^{T} & 0 \\
0 & P_{k+1}^{1 / 2} & 0 \\
0 & 0 & 0
\end{array}\right]^{+}
\end{aligned}
$$


As can be seen, the last (block) row of the left-hand side matrix in (24) is zero. Thus, the last (block) row of the matrix $\partial_{\theta_{i}} Q_{k} \cdot Q_{k}^{T}$ must exactly cancel the last (block) row of the second term in 24):

$$
\left[\partial_{\theta_{i}} Q_{k} \cdot Q_{k}^{T}\right]_{\text {col: } 1: m+n}^{\text {row: last } q}=-\left[\begin{array}{ll}
B_{i} & K_{i}
\end{array}\right]\left[\begin{array}{cc}
R_{e, k}^{1 / 2} & \bar{K}_{p, k}^{T} \\
0 & P_{k+1}^{1 / 2}
\end{array}\right]^{-1} .
$$

By substituting 25] into 23, we obtain

$$
\left[\partial_{\theta_{i}} Q_{k} \cdot Q_{k}^{T}\right]_{\text {col: last } q}^{\text {row: } 1: m+n}=\left[\begin{array}{cc}
R_{e, k}^{1 / 2} \bar{K}_{p, k}^{T} \\
0 & P_{k+1}^{1 / 2}
\end{array}\right]^{-T}\left[\begin{array}{c}
B_{i} \\
K_{i}
\end{array}\right] .
$$

Final substitution of (26) into (22) validates (10) of the proposed method for the Log LG evaluation. This completes the proof.

Remark 1: The method for score evaluation introduced above has been derived from the eSRCF implementation. As a consequence, the proposed method is of covariance-type.

Remark 2: The new square-root algorithm for score evaluation naturally extends the eSRCF filter and, hence, consists of two parts. They are the "filtered" and "differentiated" parts. This structure allows the Log LF and its gradient to be computed simultaneously. Thus, the method is ideal for simultaneous state estimation and parameter identification.

Remark 3: In the KF formulation of the Log LG evaluation, it is necessary to run the "differentiated" KF for each of the parameters $\theta_{i}$ to be estimated. As in [10], in the eSRCF formulation this "bank" of filters is replaced with the augmented arrays to which orthogonal transformations are applied.

\section{NUMERICAL RESULTS}

First, we would like to check our theoretical derivations. To do so, we apply the square-root algorithm introduced in Theorem 1 to the following simple test problem.

Example 1: Consider the special case of the system (1), (2) being

$x_{k}=\left[\begin{array}{l}d_{k} \\ s_{k}\end{array}\right]=\left[\begin{array}{cc}1 & \Delta t \\ 0 & e^{-\Delta t / \tau}\end{array}\right] x_{k-1}+\left[\begin{array}{l}0 \\ 1\end{array}\right] w_{k}, z_{k}=\left[\begin{array}{ll}1 & 0\end{array}\right] x_{k}+v_{k}$ where $w_{k} \sim N\left(0, I_{2}\right), v_{k} \sim N\left(0, I_{1}\right), I_{n}$ denotes the $n \times n$ identity matrix and $\tau$ is a parameter which needs to be estimated.

In our simulation experiment, we compute the negative Log LF and its gradient by the proposed square-root method and, then, compare the results to those produced by the conventional KF approach. The outcomes of this experiments are illustrated by Fig. 1 and 2

As can be seen from Fig. 2, all algorithms for score evaluation produce exactly the same result and give the same zero point that further coincides with the minimum point of the negative Log LF (see Fig. (1). All these evidences substantiate the theoretical derivations of Section III

Next, we wish to answer the second question posed in this paper: does the algorithm for score evaluation derived from numerically stable square-root implementation method improve the robustness of computations against roundoff errors? The previously obtained results (Example 1) indicate that both methods, i.e. the conventional $\mathrm{KF}$ technique and the new square-root algorithm, produce exactly the same answer for the Log LF and Log LG evaluation. However, numerically they no longer agree. We are now going to explore the accuracy of the numerical algorithms.

To begin designing the ill-conditioned test problem we, first, stress the type of the proposed method. As discussed in Remark 1, the new square-root algorithm belongs to the class of covariance-type methods. From Verhaegen and Van Dooren's celebrated paper [6], we know that the condition number of the innovation covariance matrix $K\left(R_{e, k}\right)$ is the key parameter determining the numerical behavior of

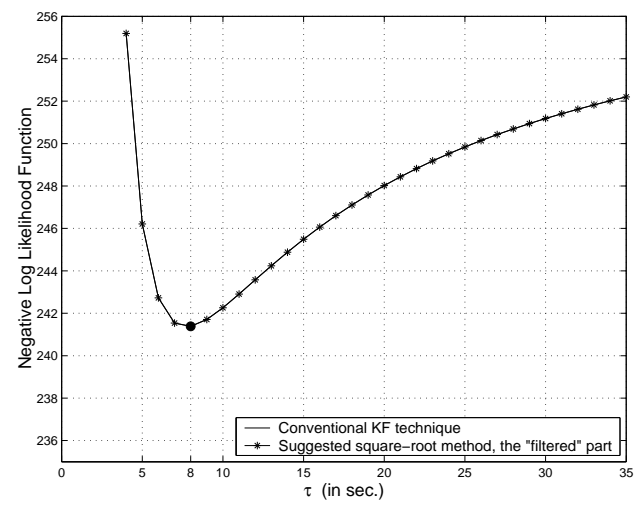

Fig. 1. The negative Log LF computed by the eSRCF and the conventional $\mathrm{KF}$ for Example 1

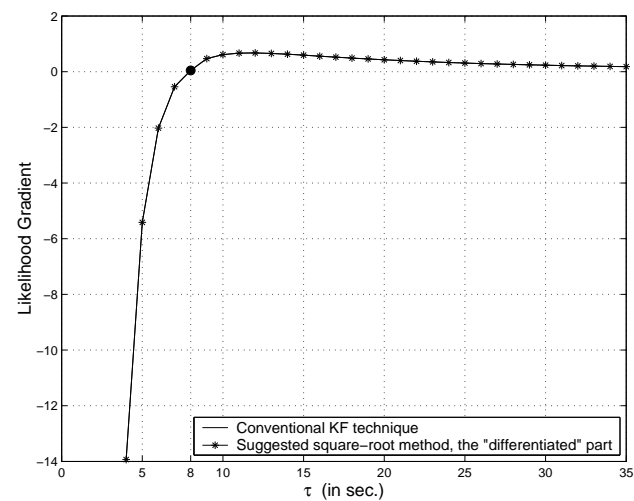

Fig. 2. The Log LG computed by the proposed square-root method and the conventional KF for Example 1

the covariance algorithms. Taking into account these two important facts, we construct the following ill-conditioned test problem.

Example 2: Consider the problem with the measurement sensitivity matrix

$$
H_{k}=\left[\begin{array}{ccc}
1 & 1 & 1 \\
1 & 1 & 1+\delta
\end{array}\right] \text { and } F_{k}=I_{3}, G_{k}=0, Q_{k}=I_{1}, R_{k}=\delta^{2} \theta I_{2}
$$

with $x_{0} \sim \mathcal{N}\left(0, \theta I_{3}\right)$, where $\theta$ is an unknown system parameter. To simulate roundoff we assume that $\delta^{2}<\epsilon_{\text {roundof }}$, but $\delta>\epsilon_{\text {roundof } f}$ where $\epsilon_{\text {roundoff }}$ denotes the unit roundoff errot

When $\theta=1$, Example 2 coincides with well-known ill-conditioned filtering problem (see, for instance, [2]) and demonstrates how a problem that is well-conditioned, as posed, can be made illconditioned by the filter implementation. The difficulty to be explored is in matrix inversion. As can be seen, although $\operatorname{rank} H=2$, the matrix $R_{e, 1}$ is singular in machine precision that yields the failure of the conventional KF implementation. We introduced an unknown system parameter $\theta$ making sure that the same problem is applied to the matrix $\left(R_{e, 1}\right)_{\theta}^{\prime}$ for each value of $\theta$. Thus, both parts of the method for score evaluation, that are the "filtered" and "differentiated" parts, fail after processing the first measurement. From the discussion above we understand that Example 2 demonstrates the difficulty only for the covariance-type methods.

Our simulation experiments presented below are organized as follows. All methods were implemented in the same precision (64-bit

${ }^{2}$ Computer roundoff for floating-point arithmetic is often characterized by a single parameter $\epsilon_{\text {roundoff } f}$, defined in different sources as the largest number such that either $1+\epsilon_{\text {roundoff }}=1$ or $1+\epsilon_{\text {roundoff }} / 2=1$ in machine precision. 
TABLE I

EFFECT OF ROUNDOFF ERRORS ON THE COMPUTED SOLUTIONS FOR THE SET OF TEST PROBLEMS FROM EXAMPLE2

\begin{tabular}{c|c||c|c|c|c||c|c|c|c}
\hline \multicolumn{2}{c||}{ Problem conditioning } & \multicolumn{4}{c||}{ Conventional KF technique } & \multicolumn{3}{c}{ Suggested square-root method } \\
\hline$\delta$ & $K\left(R_{e, 1}\right)$ & $\Delta P_{1}$ & $\Delta P_{1}^{\prime}$ & $\Delta \log L F$ & $\Delta \log L G$ & $\Delta P_{1}$ & $\Delta P_{1}^{\prime}$ & $\Delta \log L F$ & $\Delta \log L G$ \\
\hline $10^{-2}$ & $10^{3}$ & $1 \cdot 10^{-13}$ & $1 \cdot 10^{-10}$ & $2 \cdot 10^{-13}$ & $1 \cdot 10^{-13}$ & $4 \cdot 10^{-15}$ & $7 \cdot 10^{-16}$ & $1 \cdot 10^{-13}$ & $9 \cdot 10^{-14}$ \\
$10^{-4}$ & $10^{7}$ & $5 \cdot 10^{-10}$ & $9 \cdot 10^{-4}$ & $4 \cdot 10^{-9}$ & $1 \cdot 10^{-9}$ & $4 \cdot 10^{-13}$ & $7 \cdot 10^{-14}$ & $6 \cdot 10^{-10}$ & $7 \cdot 10^{-10}$ \\
$10^{-6}$ & $10^{11}$ & $2 \cdot 10^{-6}$ & $2 \cdot 10^{-1}$ & $2 \cdot 10^{-5}$ & $6 \cdot 10^{-6}$ & $3 \cdot 10^{-11}$ & $1 \cdot 10^{-11}$ & $9 \cdot 10^{-6}$ & $4 \cdot 10^{-6}$ \\
$10^{-8}$ & $10^{15}$ & $3 \cdot 10^{-3}$ & $2 \cdot 10^{-1}$ & $3 \cdot 10^{-1}$ & $2 \cdot 10^{-2}$ & $3 \cdot 10^{-10}$ & $2 \cdot 10^{-10}$ & $2 \cdot 10^{-1}$ & $9 \cdot 10^{-3}$ \\
$10^{-9}$ & $10^{16}$ & $3 \cdot 10^{-1}$ & $\mathrm{NaN}$ & $4 \cdot 10^{0}$ & $4 \cdot 10^{0}$ & $2 \cdot 10^{-8}$ & $7 \cdot 10^{-9}$ & $1 \cdot 10^{0}$ & $5 \cdot 10^{1}$ \\
$10^{-10}$ & $\infty$ & $\mathrm{NaN}$ & $\mathrm{NaN}$ & $\mathrm{NaN}$ & $\mathrm{NaN}$ & $2 \cdot 10^{-7}$ & $1 \cdot 10^{-8}$ & $2 \cdot 10^{4}$ & $2 \cdot 10^{4}$ \\
\hline
\end{tabular}

floating point) in MatLab where the unit roundoff error is $2^{-53} \approx$ $1.11 \cdot 10^{-16}$. The MatLab function eps is twice the unit roundoff error and $\delta=e p s^{2 / 3}$ satisfies the conditions $\delta^{2}<\epsilon_{\text {roundoff }}$ and $\delta>\epsilon_{\text {roundoff }}$ from Example 2 We provide the computations for for different values of $\delta$, say $\delta \in\left[10^{-9} \mathrm{eps}^{2 / 3}, 10^{9} \mathrm{eps}^{2 / 3}\right]$. This means that we consider a set of test problems from Example 2 The unknown system parameter $\theta$ is fixed, say $\theta=2$. The exact answers are produced by the Symbolic Math Toolbox of MatLab.

Experiment 1: In this experiment we are going to use the performance profile technique to compare the conventional $\mathrm{KF}$ approach for score evaluation with the square-root algorithm introduced in this paper. The performance profile method was developed by Dolan and Moré [12] to answer a common question in scientific computing: how to compare several competing methods on a set of test problem. Now, it can be found in textbooks (see, for instance, [13]).

In our simulation experiments we consider a set $A$ of $n=2$ algorithms, mentioned above. The performance measure, $t_{a}(p)$, is a measure of accuracy. More precisely, $t_{a}(p)$ is the maximum absolute error in Log LG computed for 7 different values of $\delta$. Thus, we consider a set $P$ of $m=7$ test problems from Example 2. $\delta \in\left[10^{-2}, 10^{-3}, 10^{-4}, 10^{-5}, 10^{-6}, 10^{-7}, 10^{-8}\right]$. According to the performance profile technique, we compute the performance ratio

$$
r_{p, a}=\frac{t_{a}(p)}{\min \left\{t_{\sigma}(p): \sigma \in A\right\}} \geq 1,
$$

which is the performance of algorithm $a$ on problem $p$ divided by the best performance of all the methods (we mean a particular implementation method for score evaluation) on this problem. The performance profile of algorithm $a$ is the function

$$
\phi_{a}(\mu)=\frac{1}{m} \times \text { number of } p \in P \text { such that } r_{p, a} \leq \mu,
$$

which is monotonically increasing. Thus, $\phi_{a}(\mu)$ is the probability that the performance of algorithm $a$ is within a factor $\mu$ of the best performance over all implementations on the given set of problems.

The results of this experiment are illustrated by Fig. 3 For each method, $\mu$ is plotted against the performance profile $\phi_{a}(\mu)$, for $\mu \in$ $[0,3]$. We are now going to explain Fig. 3

Let us consider the left-hand side of Fig. 3 where $\mu=1$. We can say that the new square-root algorithm proposed in this paper is the most accurate implementation on $\approx 71 \%$ of the problems, with the conventional KF being accurate on $30 \%$ of the problems. Next, we consider the middle of the plot, looking where the curve first hit probability 1 . We conclude that the suggested square-root method is within a factor $\mu \approx 1.3$ of being the most accurate implementation on every test problem. However, the conventional KF approach for score evaluation will never manage all 7 problems (as $\delta \rightarrow \epsilon_{\text {roundoff } f}$, the machine precision limit, the test problems become ill-conditioned). We need to increase $\mu$ to $\approx 2.7$ to be able to say that for $\approx 58 \%$ of the test problems the conventional KF provides an accurate Log LG evaluation within a factor $\mu \approx 2.7$.

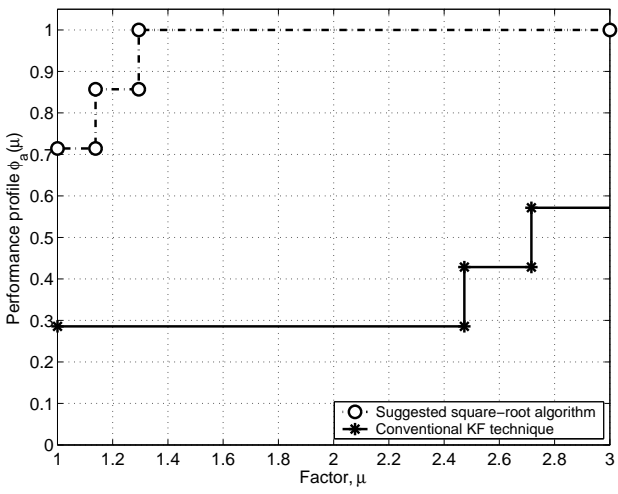

Fig. 3. Performance profiles of the methods for score evaluation: the conventional KF implementation and the new square-root algorithm proposed in this paper, - on the set of test problems from for Example 2

Thus, the performance profiles clearly indicate that on the set of the test problems from Example 2 the new square-root algorithm derived in this paper provides more accurate evaluation of the Log LG compared with the conventional KF approach.

Experiment 2: In this experiment we use the conventional KF technique and the proposed square-root method to compute the maximum absolute error in $\log \mathrm{LF}$, denoted as $\triangle \log L F$, and its gradient, denoted as $\Delta \log L G$. The results of this experiment are summarized in Table \ We also present the maximum absolute error among elements in matrices $P_{1}$ and $\left(P_{1}\right)_{\theta}^{\prime}$ (denoted as $\Delta P_{1}$ and $\Delta P_{1}^{\prime}$, respectively) to explore the numerical behavior of the "filtered" and "differentiated" parts of the methods for score evaluation.

As can be seen from Table [ the square-root implementation of the Riccati-type sensitivity equation degrades more slowly than the conventional Riccati-type sensitivity recursion as $\delta \rightarrow \epsilon_{\text {roundoff }}$, the machine precision limit (see columns denoted as $\Delta P_{1}^{\prime}$ ). For instance, the "filtered" (columns $\Delta P_{1}$ ) and "differentiated" (columns $\left.\Delta P_{1}^{\prime}\right)$ parts of the proposed square-root method for score evaluation maintain about 7 and 8 digits of accuracy, respectively, at $\delta=10^{-9}$. The conventional KF technique provides essentially no correct digits in both computed solutions. Besides, it seems that the roundoff errors tend to accumulate and degrade the accuracies of the Log LF and Log LG faster than the accuracies of $\Delta P_{1}$ and $\Delta P_{1}^{\prime}$. Indeed, for the same $\delta=10^{-9}$ we obtain no correct digits in the computed solutions for all methods. In MatLab, the term 'NaN' stands for 'Not a Number' that actually means the failure of the numerical algorithm.

Remark 4: The results of Experiment 2 indicate that the new square-root algorithm provides more accurate computation of the sensitivity matrix $\left(P_{k}\right)_{\theta}^{\prime}$ compared to the conventional KF. Hence, it can be successfully used in all applications where this quantity is required. 


\section{CONCLUding REMARKS}

In this paper, a numerically stable square-root implementation method for KF formulas, the eSRCF, has been extended in order to compute the Log LG for linear discrete-time stochastic systems. The preliminary analysis indicates that the new algorithm for score evaluation provides more accurate computations compared with the conventional KF approach. The new result can be used for efficient calculations in sensitivity analysis and in gradient-search optimization algorithms for the maximum likelihood estimation of unknown system parameters.

As an extension of the eSRCF, the new method for score evaluation is expected to inherit its benefits. However, the question about suitability for parallel implementation is still open.

It can be mentioned that another approach to construct numerically stable implementation method for score evaluation is to use the $U D$ filter [3]. Being the modification of the square-root implementations, the $U D$-type algorithms improve the robustness of computations against roundoff errors, but compared with SRF, the $U D$ filter reduces the computational cost (see [3], [6], [5]). As mentioned in [10] and as far as this author knows, it is still not known how to use the $U D$ filter to compute the score.

\section{REFERENCES}

[1] F.C. Schweppe, "Evaluation of likelihood functions for Gaussian signals", IEEE Trans. Inf. Theory, vol. IT-11, pp. 61-70, Jan. 1965.

[2] M.S. Grewal and A.P. Andrews, Kalman Filtering: Theory and Practice Using MATLAB, 2nd ed., Ed. New York: John Wiley \& Sons, 2001.

[3] G.J. Bierman, Factorization Methods for Discrete Sequential Estimation. Ed. New York: Academic Press, 1977.

[4] P.G. Kaminski, A.E. Bryson and S.F. Schmidt, "Discrete square-root filtering: a survey of current techniques", IEEE Trans. Autom. Control, vol. AC-16, pp. 727-735, Dec. 1971.

[5] T. Kailath, A.H. Sayed and B. Hassibi, Linear Estimation, Ed. New Jersey: Prentice Hall, 2000.

[6] M. Verhaegen and P.V. Dooren, "Numerical aspects of different Kalman filter implementations", IEEE Trans. Autom. Control, vol. AC-31, pp. 907-917, Oct. 1986.

[7] T. Kailath, "Array algorithms for structured matrices", presented at the conference of the International Linear Algebra Society, Winnipeg, USA, June 3-6, 1998.

[8] P. Park and T. Kailath, "New square-root algorithms for Kalman filtering", IEEE Trans. Autom. Control, vol. 40, pp. 895-899, May 1995.

[9] E.K.B. Lee and S. Maykin, "Parallel implementation of the extended square-root covariance filter for tracking applications", IEEE Trans. Parallel Distrib. Syst., vol. 4, pp. 446-457, April 1993.

[10] G.J. Bierman, M.R. Belzer, J.S. Vandergraft and D.W. Porter, "Maximum likelihood estimation using square root information filters", IEEE Trans. Autom. Control, vol. 35, pp. 1293-1298, Dec. 1990.

[11] P. Dyer and S. McReynolds, "Extensions of square root filtering to include process noise", J. Opt. Theory Appl., vol. 3, pp. 444-459, June 1969.

[12] E.D. Dolan and J.J. Moré, "Benchmarking optimization software with performance profiles", Math. Programming, vol. 91:201-213, pp. 320348, 2002.

[13] D.J. Higham and N.J. Higham, MatLab Guide, 2nd ed., Ed. Philadelphia: SIAM, 2005. 